\title{
Bacterial diversity of symptomatic primary endodontic infection by clonal analysis
}

Letícia Maria Menezes NÓBREGA(a) Francisco MONTAGNER R $^{(b)}$ Adriana Costa RIBEIRO(c) Márcia Alves Pinto MAYER ${ }^{(c)}$ Brenda Paula Figueiredo de Almeida GOMES(a)

(a) Universidade Estadual de Campinas (UNICAMP), Piracicaba Dental School, Endodontics Division, Piracicaba, SP, Brazil.

(b) Universidade Federal do Rio Grande do Sul (UFRGS), Department of Conservative Dentistry, Porto Alegre, RS, Brazil.

(c) Universidade de São Paulo (USP), Institute of Biomedical Science, Department of Oral Microbiology, São Paulo, SP, Brazil.

Declaration of Interests: The authors certify that they have no commercial or associative interest that represents a conflict of interest in connection with the manuscript.

Corresponding Author: Brenda P. F. A. Gomes E-mail: bpgomes@fop.unicamp.br

DOI: 10.1590/1807-3107BOR-2016.vol30.0103

Submitted: Dec 17, 2015

Accepted for publication: Mar 23, 2016

Last revision: Jul 05, 2016
Abstract: The aim of this study was to explore the bacterial diversity of 10 root canals with acute apical abscess using clonal analysis. Samples were collected from 10 patients and submitted to bacterial DNA isolation, 16S rRNA gene amplification, cloning, and sequencing. A bacterial genomic library was constructed and bacterial diversity was estimated. The mean number of taxa per canal was 15 , ranging from 11 to 21 . A total of 689 clones were analyzed and 76 phylotypes identified, of which 47 (61.84\%) were different species and 29 (38.15\%) were taxa reported as yet-uncultivable or as yet-uncharacterized species. Prevotella spp., Fusobacterium nucleatum, Filifactor alocis, and Peptostreptococcus stomatis were the most frequently detected species, followed by Dialister invisus, Phocaeicola abscessus, the uncharacterized Lachnospiraceae oral clone, Porphyromonas spp., and Parvimonas micra. Eight phyla were detected and the most frequently identified taxa belonged to the phylum Firmicutes (43.5\%), followed by Bacteroidetes (22.5\%) and Proteobacteria (13.2\%). No species was detected in all studied samples and some species were identified in only one case. It was concluded that acute primary endodontic infection is characterized by wide bacterial diversity and a high intersubject variability was observed. Anaerobic Gram-negative bacteria belonging to the phylum Firmicutes, followed by Bacteroidetes, were the most frequently detected microorganisms.

Keywords: Endodontics; Microbiology; Cloning, Molecular.

\section{Introduction}

Acute endodontic infections are one of the most frequently treated conditions in emergency endodontic procedures. Clinically, these infections are characterized by spontaneous pain, tenderness to percussion, and pain on palpation. Microorganisms might reach the apical connective tissues, leading to an intense inflammatory response. ${ }^{1,2}$ If untreated, these infections might progress resulting in life-threatening conditions and systemic complications. The microbiota associated with acute endodontic infections is highly heterogeneous, presenting different bacterial profiles among the patients. ${ }^{1}$ Elucidating the microbial ecology of endodontic infections is a necessary step in understanding the necessity of microbial control during all phases of endodontic treatment. ${ }^{3}$

Currently, over 1,000 bacterial species belonging to 13 phylotypes have already been identified in the oral microbiota and oral cavity. ${ }^{4}$ 
These species are able to reach the root canal system and are potential endodontic pathogens. ${ }^{5}$ Nowadays, nearly 916 species have been found within the root canal system. Of these species, 486 were found in acute endodontic infections, 265 in chronic infections, and 165 in both cases. ${ }^{6}$ A limited number of species has been consistently isolated from endodontic infections due to the limitations of culture-dependent methods, since approximately $50 \%$ of the oral microbiota is still uncultivable. ${ }^{7}$ Thus, the role of more fastidious or still uncultivable microorganisms in the pathogenesis and symptomatology of primary endodontic infections or persistent periradicular lesions may have been underestimated. ${ }^{8,9,10,11}$

Molecular methods for bacterial identification based on 16S rRNA sequencing represent an important tool for both identification of cultivable and uncultivable pathogens and determination of their taxonomic position. The 16S rRNA gene has sufficiently conserved regions for accurate alignment and enough variation for phylogenetic analysis., ${ }^{32,13,14}$ Direct amplification of $16 S$ rDNA genes from DNA extracted from bacteria in clinical samples, followed by cloning and sequencing of the genes, has allowed bacterial communities to be characterized in their entirety, without the biases of culture-based studies. ${ }^{8}$

Assessment of the human oral microbiome using metagenomic approaches, with genetic material recovered from oral cavity samples, has revealed that these techniques are sensitive and precise for evaluation of bacterial diversity in root canal infections, allowing the identification of some unexpected pathogens or those not often associated with endodontic infection. ${ }^{11,15,16,17}$ Moreover, $16 \mathrm{~S}$ rDNA sequence analysis has enabled the detection of bacteria when cultures yield negative results, thereby permitting the identification of novel species in relatively small sets of samples. ${ }^{10,18}$

In spite of the disseminated use of broad-range molecular methods for bacterial identification and detection, studies using clonal analysis and sequencing of endodontic microbiota have usually evaluated a small number of samples ${ }^{10,18}$ or have investigated samples collected only from asymptomatic endodontic infection. ${ }^{16}$ Therefore, the aim of this study was to investigate the bacterial diversity of root canals with acute apical abscess.

\section{Methodology}

\section{Patient selection and clinical examination}

Samples were collected from 10 patients who sought the Piracicaba Dental School, State University of Campinas (UNICAMP), for endodontic treatment due to spontaneous pain associated with root canal infections. The study was approved by the local Research and Ethics Committee, and all patients signed an informed consent form.

The selection of patients was determined by their dental records as well as by clinical and radiographic examinations. Pulp necrosis, spontaneous pain, periapical lesion, and tenderness to percussion in the examined tooth were the inclusion criteria. The exclusion criteria comprised those patients who had received antibiotic therapy in the last 3 months and/or presented pathology associated with periodontal disease. Eight single-rooted and two multi-rooted teeth were analyzed.

\section{Sampling procedures}

Root canals were sampled as previously described by Jacinto et al. ${ }^{18}$ and Gomes et al. ${ }^{19}$ Aseptic techniques were used throughout the endodontic sample acquisition. The sampling process was performed as follows: dental caries and existing restorations were removed without exposing the canals; the tooth was isolated from the oral cavity with a rubber dam; the operatory field was then disinfected with $30 \%$ hydrogen peroxide followed by $2.5 \%$ sodium hypochlorite, which was inactivated with $5 \%$ sodium thiosulfate; and the root canals were exposed by using sterile burs under manual irrigation with sterile saline solution.

Sampling included only one root canal for each tooth. If the tooth was multi-rooted, either the largest canal in the root or the one with periradicular radiolucency was sampled in order to confine the microbial evaluation to a single environmental site. A sterile paper point was introduced into the full length of the root canal, as determined 
on a preoperative radiograph, and kept in place for 60 seconds. In the case of a dry root canal, a second paper point, moistened with sterile saline solution, was used to ensure adequate sample acquisition. In the case of a wet root canal, as many paper points as needed were used to absorb all fluid from inside the canal. The paper points were immediately transferred to a test tube containing $1 \mathrm{~mL}$ of Viability Medium Göteborg Anaerobic III (VMGA III) transport medium.

\section{DNA extraction and universal PCR amplification}

Tubes containing the samples were shaken for 60 seconds and then $300 \mu \mathrm{L}$ of the transport medium was used for DNA extraction with QIAamp DNA Minikit (Qiagen, Valencia, CA, USA), according to the manufacturer's instructions.

The universal amplification of the 16S rRNA gene was performed by means of polymerase chain reaction (PCR) in a total volume of $50 \mu \mathrm{L}$ containing $5 \mu \mathrm{L}$ of template DNA, $5 \mu \mathrm{L}$ of 10X PCR buffer, $1.5 \mu \mathrm{L}$ of $25 \mathrm{mmol} / \mathrm{L} \mathrm{MgCl}_{2}, 4.0 \mu \mathrm{L}$ of dNTP solutions ( $25 \mathrm{mmol} / \mathrm{L}$ each), $1 \mu \mathrm{L}$ of $25 \mathrm{pmol}$ forward universal primer (D88 - 5' GAGAGTTTGATYMTGGCTCAG $\left.3^{\prime}\right)$ and reverse universal primer (E94 - 5' GAAGGAGGTGWTCCARCCGCA 3'), $0.5 \mu \mathrm{L}$ of $5 \mathrm{U} / \mathrm{mL}$ of platinum Taq polymerase, and sterile distilled water to a $50 \mu \mathrm{L}$ final volume. ${ }^{20}$ The reagents were synthesized and provided by Invitrogen (Carlsbad, CA, USA). The genomic DNA of $P$. gingivalis (ATCC 33277) and sterile distilled water were used as positive and negative controls, respectively. The reaction was performed in a DNA thermocycler (GenePro, Bioer Technology, Hangzhou, China) adjusted to initial denaturation step at $94^{\circ} \mathrm{C}$ for 4 minutes, followed by 30 cycles at $94^{\circ} \mathrm{C}$ for 45 seconds, $60^{\circ} \mathrm{C}$ for 45 seconds, $72^{\circ} \mathrm{C}$ for 90 seconds, and a final step at $72^{\circ} \mathrm{C}$ for 15 minutes.

Three independent $P C R$ reactions were performed for each sample. The 1500-bp fragments were revealed after agarose gel electrophoresis at $1 \%$, stained with $0.5 \mu \mathrm{L} / \mathrm{ml}$ ethidium bromide and purified by using the QIAquick Gel Extraction kit (Qiagen, North Rhine-Westphalia, Germany) according to the manufacturer's instructions.

\section{Clonal analysis and 16S rRNA sequencing}

The 16S rRNA gene products were ligated into the pCR2.1-TOPO vector (TOPO TA Cloning Kit - Invitrogen, Carlsbad, California, USA) and inserted into electrocompetent DH5a Escherichia coli by means of electroporation (MicroPulser, Bio-Rad Laboratories, Hercules, USA). The transformed cells were plated onto Luria-Bertani agar plates supplemented with ampicillin $(100 \mathrm{mg} / \mathrm{ml})$ and X-Gal (Promega), and then incubated overnight. Ninety-six white colonies per sample were randomly selected, suspended in $40 \mu \mathrm{L}$ of $10 \mathrm{mmol} / \mathrm{L}$ Tris-EDTA, and used as template $(5 \mu \mathrm{L})$ to determine the correct sizes of the inserts by PCR. The amplification reaction was performed with M13 forward (5' GTAAAACGACGGCCAG 3') and M13 reverse (5' CAGGAAACAGCTATGAC 3') primers. The cycling conditions comprised initial denaturation at $94^{\circ} \mathrm{C}$ for 10 minutes and included 30 cycles at $94^{\circ} \mathrm{C}$ for 45 seconds, $60^{\circ} \mathrm{C}$ for 45 seconds, and $72^{\circ} \mathrm{C}$ for 90 seconds, followed by a final extension at $72^{\circ} \mathrm{C}$ for 10 minutes. The PCR products (approximately 1,500 bp) were submitted to agarose gel electrophoresis at $1 \%$ and purified using the GTX PCR DNA Purification kit (GE Healthcare, Chalfont St Giles, Buckinghamshire, UK) before sequencing.

The concentrations of purified PCR products were verified by agarose gel electrophoresis at $2 \%$ by using a low-mass DNA ladder. The purified PCR products at a concentration of approximately $40 \mathrm{ng} / \mu \mathrm{L}$ were sequenced by using the ABI 3730 DNA analyzer, with the BigDye Terminator Cycle Sequencing Kit (Applied Biosystems, Foster City, CA, USA) using primer 533R (5' TKACCGCGGCTGCTG 3'). ${ }^{16}$

\section{Data analysis}

Sequences with more than four ambiguous characters were discarded. Sequences of approximately 500-700 bases were obtained from each amplicon, and the identity/approximate phylogenetic position was obtained by comparison with 16S rRNA gene sequences from the GenBank database (www.ncbi. nlmnih.gov) through the basic local alignment search tool (BLAST) algorithm considering a 98\% similarity level. 
The Bionumerics software (Applied Maths, Saint-Martens-Latem, Belgium) was used for data entry, editing, sequence alignment, structure comparison, secondary structure comparison, similarity matrix generation, and phylogenetic tree construction. The similarity matrices were corrected for multiple base changes at a single position by the method of Jukes and Cantor. Phylogenetic trees were constructed by using unweighted pair group method with arithmetic mean (UPGMA). ${ }^{16}$

\section{Results}

A total of 799 cloned 16S rRNA fragments were sequenced. Sequences with more than four ambiguous characters or fragment length shorter than $500 \mathrm{bp}$ were discarded, resulting in 689 appropriate sequences, which were used for the phylogenetic analysis.

The number of clones analyzed in each case ranged from 55 to 86 , depending on the fragment sequence quality. The proportion of each phylotype was estimated considering the microbiota recovered in each case. Most of the species and phylotypes identified in this study represented less than $10 \%$ of the total microbiota recovered from each sample. However, some species accounted for more than $30 \%$ of the microbiota per sample.

The mean number of bacterial taxa per canal ranged from 11 to 21, most of which consisted of anaerobic Gram-negative bacteria. The phylogenetic tree, built upon the $16 \mathrm{~S}$ rRNA gene sequence comparison of 1,500 aligned bases (Jukes and Cantor's method), is shown in Figure 1.

Prevotella oris represented more than $10 \%$ of the total microbiota and was recovered in Cases 4 and 5 . The other species of this genus represented less than $10 \%$. Phocaeicola abscessus represented more than $30 \%$ of the microbiota recovered in Case 10. Moreover, species recovered from a single sample, such as Exiguobacterium mexicanum, Enterococcus faecalis, and Streptococcus intermedius, were found in high proportion (over 30\%) in these samples. Veilonella parvula, Micrococcus flavus, Acinetobacter lwoffi, and Paracoccus sp. represented more than $10 \%$ of the microbiota of the samples that were positive for these species (Figure 1).
The most frequently detected taxa belonged to the phylum Firmicutes (43.5\% of the identified taxa), identified in all 10 samples, followed by the phylum Bacteroidetes (22.5\%), detected in nine samples. The phylum Fusobacteria represented only $3.9 \%$ of the identified taxa; however, it was present in seven samples. The phylum Proteobacteria (13.2\%) was detected in five samples, Actinobacteria (6.5\%) in four samples, Synergistetes (5.2\%) and Spirochaetes $(3.9 \%)$ in two samples, and Deinococcus-Thermus $(1.3 \%)$ in one sample. There was no case harboring the eight detected phyla. A high intersubject variability was observed. For example, Case 6 presented only Firmicutes and Bacteroidetes in a total of 11 phylotypes, whereas Case 8 presented Firmicutes, Bacteroidetes, Proteobacteria, Fusobacteria, Spirochaetes, and Synergistetes in a total of 21 different phylotypes.

Seventy-six phylotypes were identified in this study, of which 47 (61.84\%) were different species and $29(38.15 \%)$ were taxa reported as yet-uncultivable or yet-uncharacterized species. Forty-three phylotypes were present in only one sample.

Species belonging to the genus Prevotella, including P. baroniae, P. buccae, P. denticola, P. intermedia, P. loescheii, P. maculosa, P. marshii, P. oris, and P. oulorum were detected in nine out of the 10 samples studied. Fusobacterium nucleatum, Filifactor alocis, and Peptostreptococcus stomatis were the most frequently detected species (6/10 canals), followed by Dialister invisus, Phocaeicola abscessus, Eubacterium spp., the uncharacterized Lachnospiraceae oral clone (5/10 canals), Porphyromonas spp., and Parvimonas micra (4/10 canals). Figure 2 shows the number of cases where the most frequently bacteria were detected.

\section{Discussion}

Open-ended molecular approaches that use 165 rRNA analysis, such as cloning and sequencing of PCR products from polymicrobial infections, allow assessing of predicting the general microbiota, involving fastidious, slow-growing or yet-uncultivable bacteria, without searching for target species only. Usually, the initial 500-bp sequence of the 16S rRNA gene allows adequate differentiation and is sufficient for the identification of clinical isolates. ${ }^{13,14}$ 
16sRrma. 1500

$\begin{array}{llllllllllllllll}70 & 72 & 74 & 76 & 78 & 80 & 82 & 84 & 86 & 88 & 90 & 92 & 94 & 96 & 98 & 100\end{array}$

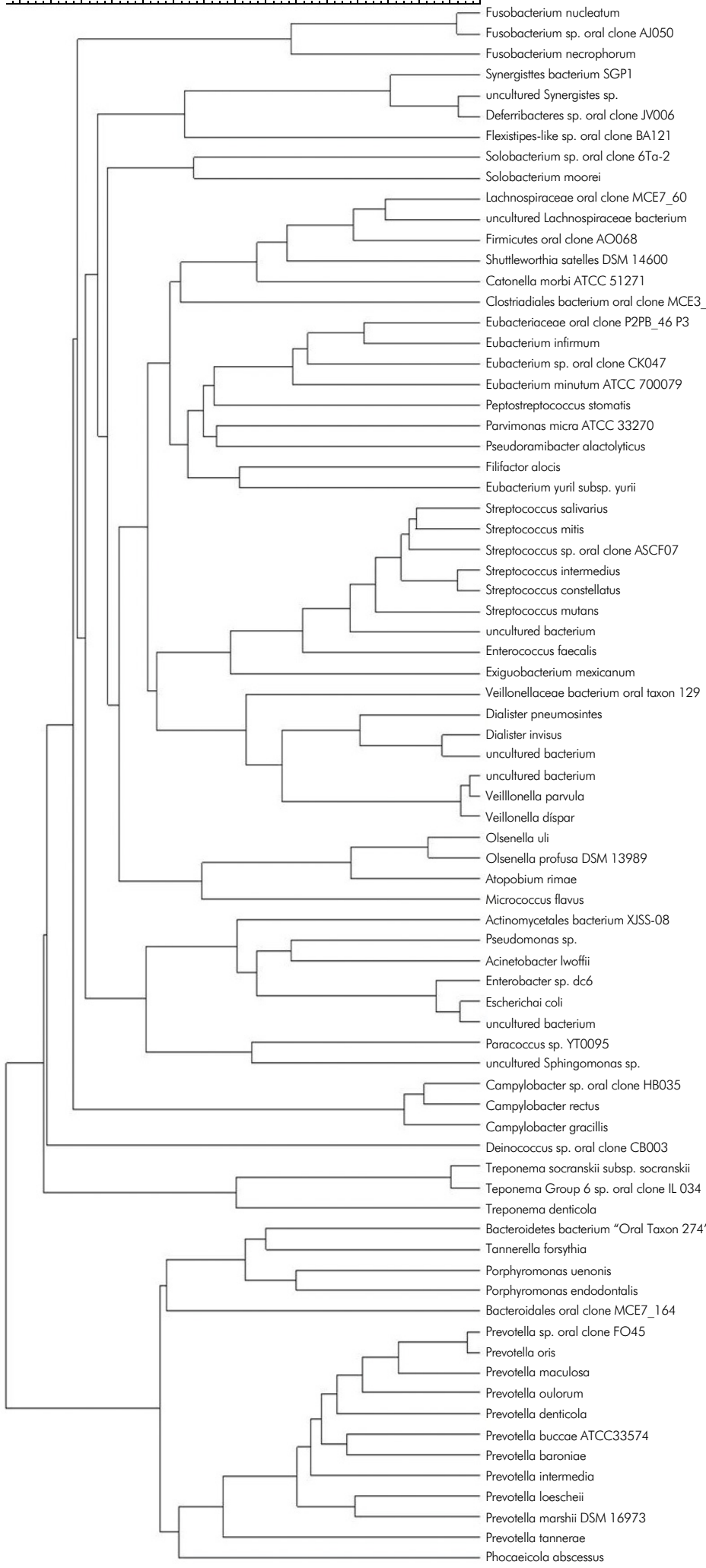

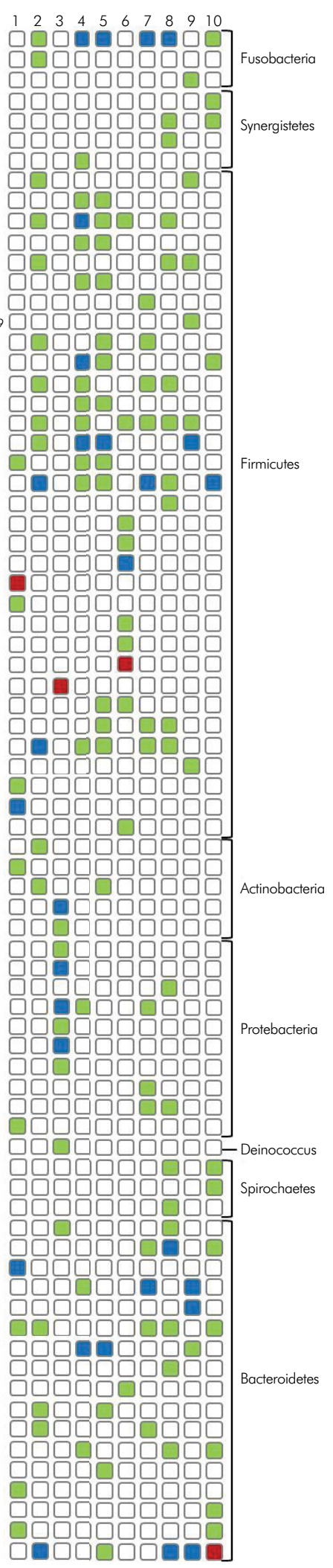

Figure 1. Phylogenetic tree based on $16 \mathrm{~S}$ rRNA gene sequence $(1,500 \mathrm{bp})$. Scale bar represents percentage sequence similarity. The column of boxes represents the bacterial profile of each patient $(n=10)$ and proportion is indicated by the following colors: white (absence), green $(<10 \%)$, blue $(10 \%-30 \%)$, and red $(>30 \%)$. 


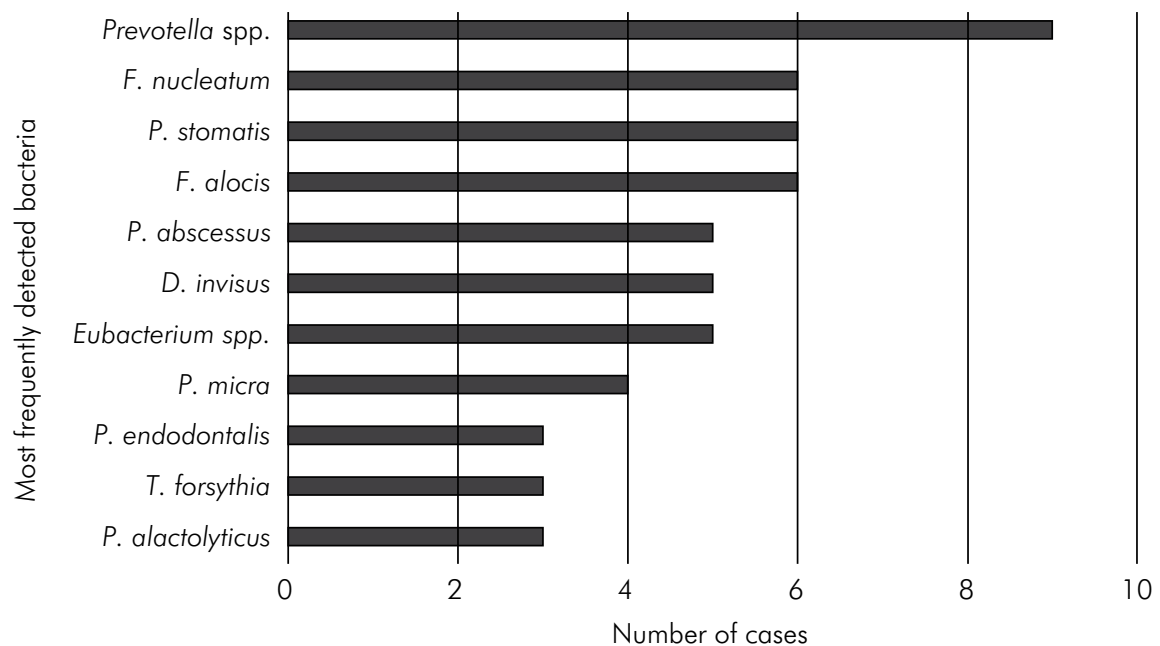

Figure 2. Number of cases presenting the most frequently identified bacteria

Cloning and sequencing do not indicate bacterial viability, which is available from assays directed towards the detection of mRNA through RT-PCR. Although it is possible to detect DNA from dead cells in endodontic infections, this possibility is conceivably low. It is highly unlikely that free bacterial DNA will remain intact in an environment colonized by living microorganisms. DNAses released by some living species and at cell death can degrade free DNA in the environment. ${ }^{15}$ Furthermore, primers designed to generate large amplicons may reduce the risks of the detection of dead cells. Thus, the species detected in the present study probably represent endodontic pathogens that play an important role in the acute infection studied.

The results of this study show that the microbiota found in the root canals of teeth with primary endodontic infection and acute apical abscess is polymicrobial, with predominance of anaerobic Gram-negative bacteria. A significant number of Gram-positive bacteria has also been detected, such as Parvimonas micra, Pseudoramibacter alactolyticus, Peptostreptococcus stomatis, and Eubacterium spp. The number of different species identified in each sample ranged from 11 to 21 , whereas culture-dependent methods showed an average of 5 to 7 species per case. .,19,21 $^{8}$

The bacterial profiles in endodontic infections varied from individual to individual. No species was present in all samples and the number of patients analyzed allowed observing higher bacterial diversity than that reported in other studies. ${ }^{8,18}$ This indicates that primary endodontic infection has a heterogeneous etiology, where no single species can be considered as the main endodontic pathogen. Multiple bacterial combinations play a role in disease etiology, acting synergistically and increasing virulence, which causes further damage to the host. ${ }^{1,12,18,19,22}$ As-yet-unknown species are also present in infectious diseases, giving rise to new concepts in the pathogenesis of several human infections and redirecting therapeutic strategies. Our results show uncultured bacteria in eight cases.

Specific oral bacteria, some of them detected in this study, such as Solobacterium moorei, Phocaeicola abscessus, Porphyromonas gingivalis, Treponema denticola, and Tannerella forsythia, have been related to several systemic diseases, including bacterial endocarditis, aspiration pneumonia, osteomyelitis, brain abscess, preterm low birth weight, cardiovascular disease, and obesity, ${ }^{23-25}$ thus emphasizing the importance of accurate microbial identification.

Bacteria detected in this research were distributed into eight phyla: Firmicutes, Bacteroidetes, Proteobacteria, Actinobacteria, Synergistetes, Fusobacteria, Spirochaetes, and Deinococcus. These data are in agreement with those provided by human microbiome, periodontal and endodontic infection studies. $3,9,11,16,20,26$

Deinococcus sp, detected in only one sample, belongs to the phylum Deinococcus-Thermus. It had been previously known to exist only in extreme 
environments and in animal feces before being recently identified in human stomach and in endodontic infection. ${ }^{17}$

Synergistetes is a recently described phylum that includes Flexistipes-like sp. and Deferribacteres sp., which had been previously misclassified as belonging to the phylum Deferribacteres, ${ }^{11,27}$ and reported as an important component of endodontic infection. ${ }^{18,26,28}$ However, Synergistetes were not detected by Vickerman et al. ${ }^{9}$ and Saito et al. ${ }^{29}$

Some important bacterial species detected in the present study, such as Fusobacterium nucleatum, Dialister spp., Prevotella spp., Porphyromonas gingivalis, Filifactor alocis, and Streptococcus spp., had already been found in endodontic infections by culture-dependent and molecular approaches, confirming their status as endodontic pathogens. ${ }^{10,18,19,21,22}$ However, our results are not in line with those reported by Munson et al. ${ }^{8}$, Saito et al., ${ }^{29}$ and Rolph et al., ${ }^{30}$ who did not find F. nucleatum, P. micra, and $P$ gingivalis using clonal analysis, probably because they investigated chronic infections. Moreover, the absence of some bacterial species in those studies might be also attributed to the intrinsic limitations of the technique or to the small sample size. Our research collected samples from 10 root canals, while Munson et al. ${ }^{8}$ and Saito et al. ${ }^{29}$ obtained their samples from five and seven root canals, respectively.

The presence of Gram-negative bacteria is important, both in terms of host inflammatory response and clinical significance, due to the presence of endotoxins (lipopolysaccharide; LPS), which are cell envelope constituents and can be secreted by cell vesicles. LPS has many biological activities, including induction of fever, cytotoxicity, fibrinolysis, and stimulation of the production of bradykinin, a potent pain mediator. Clinical investigations have elucidated the strong correlation between higher levels of endotoxins in root canals with spontaneous pain and clinical symptomatology such as pain on palpation, tenderness to percussion, and exudation. ${ }^{31,32}$

Prevotella oris and Prevotella baroniae, newly named species, are some of the most prevalent Prevotella species in endodontic infection. ${ }^{33}$ Porphyromonas gingivalis, Tannerella forsythia, and Treponema denticola form the so-called red complex, which is significantly associated with periodontal disease severity and endodontic infection. ${ }^{34,35}$ Although T. forsythia and T. denticola were detected in Case 8, P. gingivalis was not detected in any of the investigated root canals.

Dialister spp. and Filifactor alocis, which are hardly identified by biochemical methods, and Fusobacterium nucleatum, which is frequently associated with symptomatology, $18,29,36$ were detected in $50 \%$ of the root canals. Dialister spp. are extremely small $(0.4 \mu \mathrm{m})$ and it would be interesting to speculate whether these organisms could be able to penetrate dentinal tubules. ${ }^{8}$

The occurrence of spirochetes in endodontic infection has long been obscured by the difficulties in culturing these microorganisms. ${ }^{26}$ Nowadays, molecular approaches have revealed a higher prevalence of oral Treponemas, which are abundant in subgingival samples from patients with periodontitis. ${ }^{11,20}$ In our results, Treponemas were found in only two samples and our patients were free of periodontitis, suggesting the absence of cross-contamination from periodontal pockets, which is in agreement with Ribeiro et al. ${ }^{16}$

Phocaeicola abscessus is a Gram-negative coccobacillus that is strictly anaerobic, asaccharolytic, non-pigmented, non-spore-forming, and motile, member of the phylum Bacteroidetes. This species was isolated from brain abscess, described by Al-Masalma et al., ${ }^{25}$ and was detected in $50 \%$ of our samples, corresponding to more than $30 \%$ of the microbiota recovered in Case 10 and more than $10 \%$ in Cases 2, 8, and 9. No previous study reported this species in endodontic infection, although it is very similar ( $99 \%$ of similarity) to sequences of previously described uncultured bacteria (GenBank access number AF481203 and AY005066) from endodontic infections. ${ }^{25}$

We also detected a great number of Gram-positive bacteria, such as Parvimonas micra, Peptostreptococcus stomatis, Pseudoramibacter alactolyticus, and Eubacterium spp. Their cell walls contain peptidoglycans and lipoteichoic acid, which are also capable of influencing inflammatory reactions and enhancing the pathogenicity of anaerobic Gram-negative bacteria, mainly black pigmented ones. ${ }^{19,22}$

Enterococcus faecalis, facultative Gram-positive cocci, widely associated with endodontic treatment failure, ${ }^{10}$ has also been detected in primary endodontic infection. ${ }^{18,37}$ However, in the present study, this species was detected in only one sample. 
Shuttleworthia satelles, Catonella morbi, Acinetobacter lwoffii, Micrococcus flavus, and Exiguobacterium mexicanum, species that have been recently associated with endodontic infection by molecular methods, were also detected here despite their low frequency. $8,16,18,38,39$

It has become clear from diverse studies using different microbiological techniques that microbial specificity in the etiology of apical periodontitis is relatively low. However, the fact that some species are more prevalent than others may be indicative that they are also more significant when it comes to endodontic infection and periapical lesions. Although some species present in endodontic infections are likely to be mere "bystanders," it is by and large recognized that some of the species can play at least an ecological role in the community. As a consequence, their participation in the disease process cannot be refuted. ${ }^{18,39}$

\section{References}

1. Montagner F, Gomes BPFA, Kumar PS. Molecular fingerprinting reveals the presence of unique communities associated with paired samples of root canals and acute apical abscesses. J Endod. 2010;36(9):1475-9. doi:10.1016/j.joen.2010.06.004

2. Gomes BPFA, Jacinto RC, Montagner F, Sousa ELR, Ferraz, CCR. Analysis of the antimicrobial susceptibility of anaerobic bacteria isolated from endodontic infections in Brazil during a period of nine years. J Endod. 2011;37(8):1058-62. doi:10.1016/j.joen.2011.05.015

3. Tzanetakis GN, Azcarate-Peril MA, Zachaki S, Panopoulos P, Kontakiotis EG, Madianos PN et al. Comparison of bacterial community composition of primary and persistent endodontic infections using pyrosequencing. J Endod. 2015;41(8):1226-33. doi:10.1016/j.joen.2015.03.010

4. Wade WG. The oral microbiome in health and disease. Pharmacol Res. 2013;69(1):137-43. doi:10.1016/j.phrs.2012.11.006

5. Jung IY, Choi BK, Kum KY, Roh BD, Lee SJ, Lee CY et al. Molecular epidemiology and association of putative pathogens in root canal infection. J Endod. 2000;26(10):599-604. doi:10.1097/00004770-200010000-00006

6. Santos AL, Siqueira JF Jr, Rôças IN, Jesus EC, Rosado AS, Tiedje JM. Comparing the bacterial diversity of acute and chronic dental root canal infections. PLoS One. 2011;6(11):e28088. doi:10.1371/journal.pone.0028088

7. Paster BJ, Olsen I, Aas JA, Dewhirst FE. The breadth of bacterial diversity in the human periodontal pocket and other oral sites. Periodontol 2000. 2006;42(1):80-7. doi:10.1111/j.1600-0757.2006.00174.x

\section{Conclusion}

In conclusion, the clonal analysis of acute primary endodontic infections revealed a wide diversity of species infecting the root canals. Not only species usually isolated in culture studies were detected, but also species that are difficult to culture and that have been proven by molecular approaches to be endodontic pathogens. However, the specific role played by each of these species in the endodontic bacterial community awaits further clarification.

\section{Acknowledgments}

This study was supported by the Brazilian agencies FAPESP (2009/07760-5; 2011/09047-4), CNPQ (302575/2009; 308162/2014-5), and CAPES. We would like to thank Pâmela Pontes and Ericka T Pinheiro for their technical support.

8. Munson MA, Pitt-Ford T, Chong B, Weightman A, Wade WG. Molecular and cultural analysis of the microflora associated with endodontic infections. J Dent Res. 2002;81(11):761-6. doi:10.1177/154405910208101108

9. Vickerman MM, Brossard KA, Funk DB, Jesionowski AM, Gill SR. Phylogenetic analysis of bacterial and archaeal species in symptomatic and asymptomatic endodontic infections. J Med Microbiol.2007;56:110-8. doi:10.1099/jmm.0.46835-0

10. Subramanian K, Mickel AK. Molecular analysis of persistent periradicular lesions and root ends reveals a diverse microbial profile. J Endod. 2009;35(7):950-7.

11. Dewhirst FE, Chen T, Izard J, Paster BJ, Tanner ACR, Yu WH, Lakshmanan A, Wade WG. The human oral microbiome. J Bacteriol. 2010;192:5002-17.

12. Siqueira, JF, Roças IN, Oliveira JCM, Santos KRN. Detection of putative oral pathogens in acute periradicular abscesses by $16 \mathrm{~S}$ rDNA-direct polymerase chain reaction. J Endod. 2001;27:164-7.

13. Clarridge JE 3rd. Impact of $16 \mathrm{~S}$ rRNA gene sequence analysis for identification of bacterial on clinical microbiology and infectious diseases. Clin Microbiol Rev. 2004;17:840-62

14. Turnbaugh PJ, Ley RE, Hamady M, Fraser-Liggett CM, Knight R, Gordon JI. The human microbiome project. Nature. 2007;449:804-10.

15. Siqueira JF, Roças IN. Exploiting molecular methods to explore endodontic infections: Part 1 - current molecular technologies for microbiological diagnosis. J Endod. 2005;31:411-23. 
16. Ribeiro AC, Matarazzo F, Faveri M, Zezell DM, Mayer MPA. Exploring bacterial diversity of endodontic microbiota by cloning and sequencing $16 \mathrm{~S}$ rRNA. J Endod. 2011;37:922-6.

17. Li L, Hsiao WWL, Nandakumar R, Barbuto SM, Mongoidn EF, Paster BJ, Fraser-Liggett CM, Fouad AF. Analyzing endodontic infection by deep coverage pyrosequencing. J Dent Res. 2010;89:980-4.

18. Jacinto RC, Gomes BP, Desai M, Rajendran D, Shah HN. Bacterial examination of endodontic infections by clonal analysis in concert with denaturing high performance liquid chromatography. Oral Microbiol Immunol. 2007;22:403-10.

19. Gomes BP, Pinheiro ET, Gade-Neto CR, Sousa EL, Ferraz CC, Zaia AA, Teixeira, F.B., Souza-Filho, F.J. Microbiological examination of infected dental root canals. Oral Microbiol Immunol. 2004;19:71-6.

20. Paster BJ, Boches SK, Galvin JL, Ericson RE, Lau CN, Levanos VA, Sahasrabudhe A, Dewhrist FE. Bacterial diversity in human subgingival plaque. J Bacteriol. 2001;183:3770-83.

21. Siqueira JF, Roças IN, Paiva SSM, Magalhães KM, Guimarães-Pinto T. Cultivable bacteria in infected root canal as identified by $16 \mathrm{~S}$ rRNA gene sequencing. Oral Microbiol Immunol. 2007;22:266-71.

22. Jacinto RC, Gomes BP, Ferraz CC, Zaia AA, Souza-Filho FJ. Microbiological analysis of infected root canals from symptomatic and asymptomatic teeth with periapical periodontitis and the antimicrobial susceptibility of some isolated anaerobic bacteria. Oral Microbiol Immunol. 2003;18:285-92.

23. Detry G, Pierard D, Vandoorslaer K, Wauters G, Avesani V, Glupczynski Y. Septicemia due to Solobacterium moorei in patient with multiple myeloma. Anaerobe. 2006;12:160-2.

24. Goodson JM, Groppo D, Halem S, Carpino E. Is obesity an oral disease? J Dent Res. 2009;88:519-23.

25. Al-Masalma M, Raoult D. Roux V. Phocaeicola abscessus gen. Nov., sp. Nov., an anaerobic bacterium isolated from human brain abscess sample. Int J Syst Evol Microbiol. 2009;59:2232-7.

26. Gomes BP, Berber VB, Kokaras AS, Chen T, Paster BJ. Microbiomes of endodontic-periodontal lesions before and after chemomechanical preparation. J Endod. 2015;41:1975-84. doi:10.1016/j.joen.2015.08.022

27. Siqueira JF, Roças IN. The microbiota of acute apical abscesses. J Dent Res. 2009;88:61-5.
28. Vianna ME, Conrads G, Gomes BPFA, Horz HP. Quantification and characterization of Synergistetes in endodontic infection. Oral Microbiol Immunol. 2007;22:260-5.

29. Saito D, Leonardo RT, Rodrigues JL, Tsai SM, Hofling JF, Goncalves RB. Identification of bacteria in endodontic infections by sequence analysis of $16 \mathrm{~S}$ rDNA clone libraries. J Med Microbiol. 2006;55:101-7.

30. Rolph HJ, Lennon A, Riggio MP, Saunders WP, MacKenzie D, Coldero L, Bagg J. Molecular identification of microorganisms from endodontic infection. J Clin Microbiol. 2001;39:3282-9.

31. Horiba N, Maekawa Y, Abe Y, Ito M, Matsumoto T, Nakamura H. Correlations between endotoxin and clinical symptoms or radiolucent areas in infected root canals. Oral Surg Oral Med Oral Pathol. 1991;71:492-5.

32. Jacinto RC, Gomes BPFA, Shah HN, Ferraz CC, Zaia AA, Souza-Filho FJ. Quantification of endotoxins in necrotic root canals from symptomatic and asymptomatic teeth. J Med Microbiol. 2005;54:777-83.

33. Roças IN, Siqueira JF. Prevalence of new candidate pathogens Prevotella baroniae, Prevotella multisaccharivorax and as-yet-uncultivated Bacteroidetes clone X083 in primary endodontic infection. J Endod. 2009;35:1359-62.

34. Gomes BPFA, Montagner F, Jacinto RC, Zaia AA, Ferraz CCR, Souza-Filho FJ. Polymerase chain reaction of Porphyromonas gingivalis, Treponema denticola, and Tannerella forsythia in primary endodontic infections. J Endod. 2007;33:1049-52.

35. Siqueira JF, Roças IN, Silva MG. Prevalence and clonal analysis of Porphyromonas gingivalis in primary endodontic infections. J Endod. 2008;34:1332-36.

36. Siqueira JF, Roças IN. Detection of Filifactor alocis in endodontic infections associated with different forms of periradicular disease. Oral Microbiol Immunol. 2003;18:263-5.

37. Gomes BPFA, Pinheiro ET, Sousa ELR, Jacinto RC, Zaia AA, Ferraz CCR, Souza-Filho FJ. Enterococcus faecalis in dental root canals detected by culture and by polymerase chain reaction analysis. Oral Surg Oral Med Oral Pathol Oral Radiol Endod. 2006;102:247-53.

38. Downes J, Munson MA, Radford DR, Spratt DA, Wade WG Shuttleworthia satelles gen. nov., sp. nov., isolated from the human oral cavity. Int J Syst Evol Microbiol. 2002;52:1469-75.

39. Siqueira JF, Roças IN. Catonella morbi and Granulicatella adiacens: new species in endodontic infection. Oral Surg Oral Med Oral Pathol Oral Radiol Endod. 2006;102:259-64. 